\title{
Colored scalars mediated rare charm meson decays to invisible fermions
}

\author{
Svjetlana Fajfer®* and Anja Novosel $\odot^{\dagger}$ \\ Jožef Stefan Institute, Jamova 39, 1000 Ljubljana, Slovenia and Faculty of Mathematics and Physics, \\ University of Ljubljana, Jadranska 19, 1000 Ljubljana, Slovenia
}

(Received 2 February 2021; accepted 8 June 2021; published 8 July 2021)

\begin{abstract}
We consider the effects of colored scalar mediators in decays $c \rightarrow u$ invisibles. In particular, in these processes, as invisibles, we consider right-handed fermions. The colored scalar $\bar{S}_{1} \equiv(\overline{3}, 1,-2 / 3)$, due to its coupling to weak up-quark singlets and invisible right-handed fermions $(\chi)$, is very appealing. Then, we consider $\tilde{R}_{2} \equiv(\overline{3}, 2,1 / 6)$, which as a weak doublet can couple to both up- and down-quarks and invisible fermions. We determine branching ratios for $D \rightarrow \chi \bar{\chi}, D \rightarrow \chi \bar{\chi} \gamma$ and $D \rightarrow \pi \chi \chi$ for several $\chi$ masses, using most constraining bounds.The $D^{0}-\bar{D}^{0}$ mixing gives the most robust constraint for $\bar{S}_{1}$ and $\tilde{R}_{2}$ when only one down-like quark couples to invisible fermion. When $\tilde{R}_{2}$ with two nonzero Yukawa couplings is analysed, we find that the bound on $B \rightarrow K \not E$ is very constraining. We calculate for decays mediated by $\bar{S}_{1}$ that branching ratios can be $B(D \rightarrow \chi \bar{\chi})<10^{-8}$ for $m_{\chi}=0.8 \mathrm{GeV}, B(D \rightarrow \chi \bar{\chi} \gamma) \sim 10^{-12}$ for massless $\chi$, while $B\left(D^{+} \rightarrow \pi^{+} \chi \bar{\chi}\right)$ can reach $\sim 10^{-8}$ for the low mass of $\chi$. In the second scenario with $\tilde{R}_{2}$, we obtain high suppression of these decay rates. We find that future tau-charm factories and Belle II experiments offer good opportunities to search for such processes. Both $\bar{S}_{1}$ and $\tilde{R}_{2}$ might have masses within LHC reach.
\end{abstract}

DOI: 10.1103/PhysRevD.104.015014

\section{INTRODUCTION}

Low-energy constraints of physics beyond Standard Model (BSM) are well established for down-like quarks by numerous searches in processes with hadrons containing one $b$ or/and $s$ quark. However, in the up-quark sector, searches are performed in top decays, suitable for LHC studies, while in charm hadron processes at $b$-factories or/and $\tau$-charm factories. Recently, an extensive study on $c \rightarrow u \nu \bar{\nu}$ appeared in Ref. [1], pointing out that observables which are very small in the Standard Model (SM) offer unique (null) tests of BSM physics. Namely, for charm flavor changing neutral current (FCNC) processes, severe Glashow-Iliopoulos-Maiani (GIM) suppression occurs. The decay $D^{0} \rightarrow \nu \bar{\nu}$ amplitude is helicity suppressed in the SM. The authors of [2] made a very detailed study of heavy meson decays to invisibles, assuming that the invisibles can be scalars or fermions with both helicites. They found out that in the SM branching ratio $\mathcal{B}\left(D^{0} \rightarrow \nu \bar{\nu}\right)=1.1 \times 10^{-31}$. Then the authors of [3] found that the decay width of $D^{0} \rightarrow$ invisibles in the SM is

\footnotetext{
svjetlana.fajfer@ijs.si

†anja.novosel@ijs.si
}

Published by the American Physical Society under the terms of the Creative Commons Attribution 4.0 International license. Further distribution of this work must maintain attribution to the author(s) and the published article's title, journal citation, and DOI. Funded by SCOAP . actually dominated by the contribution of $D^{0} \rightarrow \nu \bar{\nu} \nu \bar{\nu}$. These studies' main message is that SM provides no irreducible background for analysis of invisibles in decays of charm (and beauty) mesons. They also suggested [2], that in searches for a Dark Matter candidate, it might be important to investigate processes with $\chi \bar{\chi} \gamma$ in the final state, since a massless photon eliminates the helicity suppression. We also determine branching ratios for such decay modes. The authors of Ref. [1] computed the expected event rate for the charm hadron decays to a final hadronic state and neutrino/antineutrino states. They found out that in experiments like Belle II, which can reach per mile efficiencies or better, the charm hadron decays to a final state containing invisible states can be seen. In addition, future FCC-ee running colliders might be capable of measuring branching ratios of $\mathcal{O}\left(10^{-6}\right)$ down to $\mathcal{O}\left(10^{-8}\right)$, particularly in $D^{0}, D_{(s)}^{+}$and $\Lambda_{c}^{+}$decay modes. On the other hand, the Belle Collaboration already reached a bound of the branching ratio for $\mathcal{B}\left(D^{0} \rightarrow\right.$ invisibles) $\leq 9.4 \times 10^{-5}$ and the Belle II experiment is expected to improve this result by one to two orders of magnitude [4]. The other $e^{+} e^{-}$machines as BESS III are expected to improve the Belle bound to the order $10^{-6} \rightarrow 10^{-5}$ [5]. Future FCC-ee running colliders at $Z$ energies [6,7] with a significant charm production with $\mathcal{B}(Z \rightarrow c \bar{c}) \simeq 0.22$ [7] provide us with excellent tools for precision study of charm decays.

In this work we focus on the particular scenarios with colored scalars or leptoquarks as mediators of the invisible 
fermions interaction with quarks. Colored scalars carry out interactions between invisible fermions and quarks. Namely, leptoquarks usually denote the boson interacting with quarks and leptons. However, the colored scalar $\bar{S}_{1}=(\overline{3}, 1,-2 / 3)$, being an electroweak singlet with an electric charge $-2 / 3 e$, does not interact with the SM leptons and, therefore, we call it a colored scalar. Instead of using a general assumption on the lepton flavor structure form [1] and justifying the Belle bound from [8], we rely on observables coming from the $D^{0}-\bar{D}^{0}$ oscillations. In the case of the weak doublet $\tilde{R}_{2}=(\overline{3}, 2,1 / 6)$, we include constraints from other flavor processes.

The LHC experiments already searched for the colored scalars decaying to the final states containing monojet and missing energy. The CMS collaboration in Ref. [9] performed such a study giving the bound on the mass of leptoquarks. They assumed that there is only one scalar leptoquark state within mass reach of the LHC and that it decays to a light quark and a neutrino. The CMS collaboration excluded masses below $980 \mathrm{GeV}$ at the $95 \%$ confidence level in the collected data. Therefore, as an example, we use the colored scalar's mass of $1000 \mathrm{GeV}$. Constraints on the parameter space of, e.g., $\bar{S}_{1}$ scalar leptoquark can decay in monojet and missing energy, as well as to two jets, due to down-like quark couplings. In the case of $\tilde{R}_{2}$, the analysis is even more complicated since the two states $\tilde{R}_{2}^{2 / 3}$ and $\tilde{R}_{2}^{5 / 3}$ have more possibilities to decay to dijets, monojet and charged lepton, and monojet and missing energy. That will require intensive and demanding analysis, not yet being done at LHC.

Motivated by the previous works of Refs. [1,2,10-13], we investigate $c \rightarrow u \bar{\chi} \chi$ with $\chi$ being a massive $S U(2)_{L}$ singlet. The effective Lagrangian and colored scalar mediators are introduced in Sec. II. In Sec. III we describe effects of $\bar{S}_{1}$ mediator in rare charm decays, while in Sec. IV we give details of $\tilde{R}_{2}$ mediation in the same processes. Sec. V contains conclusions and outlook.

\section{COLORED SCALARS IN $c \rightarrow u \chi \bar{\chi}$}

In experimental searches, the transition $c \rightarrow u$ invisibles might be approached in processes $c \rightarrow u \mathscr{E}$ with $\not E$ being missing energy. Therefore, invisibles can be either SM neutrinos or new right-handed neutral fermions (having quantum numbers of right-handed neutrinos), or scalars/ vectors as suggested in Ref. [2]. The authors of Refs. [1,10] considered in detail the general framework of new physics (NP) in $c \rightarrow u$ invisibles, relying on $S U(2)_{L}$ invariance and data on charged lepton processes [10]. They found that these assumptions allow upper limits for branching ratios as large as few $10^{-5}$, while in the limit of lepton universality branching ratios can be as large as $10^{-6}$. To consider invisible fermions, having quantum numbers of righthanded neutrinos, we extend the effective Lagrangian

$$
\begin{aligned}
\mathcal{L}_{\mathrm{eff}}= & \sqrt{2} G_{F}\left[c^{L L}\left(\bar{u}_{L} \gamma_{\mu} c_{L}\right)\left(\bar{\nu}_{L} \gamma^{\mu} \nu_{L}^{\prime}\right)\right. \\
& +c^{R R}\left(\bar{u}_{R} \gamma_{\mu} c_{R}\right)\left(\bar{\nu}_{R} \gamma^{\mu} \nu_{R}^{\prime}\right)+c^{L R}\left(\bar{u}_{L} \gamma_{\mu} c_{L}\right)\left(\bar{\nu}_{R} \gamma^{\mu} \nu_{R}^{\prime}\right) \\
& +c^{R L}\left(\bar{u}_{R} \gamma_{\mu} c_{R}\right)\left(\bar{\nu}_{L} \gamma^{\mu} \nu_{L}^{\prime}\right)+g^{L L}\left(\bar{u}_{L} c_{R}\right)\left(\bar{\nu}_{L} \nu_{R}^{\prime}\right) \\
& +g^{R R}\left(\bar{u}_{R} c_{L}\right)\left(\bar{\nu}_{R} \nu_{L}^{\prime}\right)+g^{L R}\left(\bar{u}_{L} c_{R}\right)\left(\bar{\nu}_{R} \nu_{L}^{\prime}\right) \\
& +g^{R L}\left(\bar{u}_{R} c_{L}\right)\left(\bar{\nu}_{L} \nu_{R}^{\prime}\right)+h^{L L}\left(\bar{u}_{L} \sigma^{\mu \nu} c_{R}\right)\left(\bar{\nu}_{L} \sigma_{\mu \nu} \nu_{R}^{\prime}\right) \\
& \left.+h^{R R}\left(\bar{u}_{R} \sigma^{\mu \nu} c_{L}\right)\left(\bar{\nu}_{R} \sigma_{\mu \nu} \nu_{L}^{\prime}\right)\right]+ \text { H.c.. }
\end{aligned}
$$

In Ref. [1] right-handed massless neutrinos are considered. Also, in Ref. [13] the authors considered charm meson decays to invisible fermions, which have negligible masses. In the following, we consider massive right-handed fermions and use the notation $\nu_{R} \equiv \chi_{R}$. Following [14], we write in Table I interactions of the colored scalar $\bar{S}_{1}$ and $\tilde{R}_{2}$ with the up quarks and $\tilde{R}_{2}$ and $S_{1}$ with down quarks.

We concentrate only on colored scalar and scalar leptoquarks due to difficulties with vector leptoquarks. Namely, the simplest way to consider vector leptoquarks in an ultraviolet complete theory is when they play the role of gauge bosons. For example, $U_{1}$ is one of the gauge bosons in some of the Pati-Salam unification schemes $[15,16]$. However, other particles with masses close to $U_{1}$ with many new parameters in such theories, making it rather difficult to use without additional assumptions.

Colored scalars contributing to transition $c \rightarrow u \chi \bar{\chi}$ at the tree level, have the following Lagrangians, as already anticipated in [14]

$$
\begin{gathered}
\mathcal{L}\left(\bar{S}_{1}\right) \supset \bar{y}_{1 i j}^{R R} \bar{u}_{R}^{C i} \chi_{R}^{j} \bar{S}_{1}+\text { H.c.. } \\
\mathcal{L}\left(\tilde{R}_{2}\right) \supset\left(V \tilde{y}_{2}^{L R}\right)_{i j} \bar{u}_{L}^{i} \chi_{R}^{j} \tilde{R}_{2}^{2 / 3}+\tilde{y}_{2 i j}^{L R} \bar{d}_{L}^{i} \chi_{R}^{j} \tilde{R}_{2}^{-1 / 3}+\text { H.c.. }
\end{gathered}
$$

Here, we give only terms containing interactions of quarks with right-handed $\chi$. $V$ stands for the appropriate CabibboKobayashi-Maskawa quark mixing matrix (CKM). The $S_{1}$ scalar leptoquark, in principle, might mediate $c \rightarrow u \chi \bar{\chi}$ on the loop level, with one $W$ boson changing down-like quarks to $u$ and $c$. Such a loop process is suppressed by a loop factor $1 /\left(16 \pi^{2}\right)$ and $G_{F}$ making it negligible. Also, due to the right-handed nature of $\chi$, one can immediately

TABLE I. The colored scalars $S_{1}, \bar{S}_{1}$ and $\tilde{R}_{2}$ interactions with invisible fermions and quarks. Here we use only right-handed couplings of $S_{1}$. Indices $i, j$ refer to quark generations.

\begin{tabular}{lc}
\hline \hline Colored Scalar & Invisible fermion \\
\hline$S_{1}=(\overline{3}, 1,1 / 3)$ & $\bar{d}_{R}^{C i} \chi_{R}^{j} S_{1}$ \\
$\bar{S}_{1}=(\overline{3}, 1,-2 / 3)$ & $\bar{u}_{R}^{C i} \chi_{R}^{j} \bar{S}_{1}$ \\
$\tilde{R}_{2}=(\overline{3}, 2,1 / 6)$ & $\bar{u}_{L}^{i} \chi_{R}^{j} \tilde{R}_{2}^{2 / 3}$ \\
$\tilde{R}_{2}=(\overline{3}, 2,1 / 6)$ & $\bar{d}_{L}^{i} \chi_{R}^{j} \tilde{R}_{2}^{-1 / 3}$ \\
\hline \hline
\end{tabular}


see that in the case of $\bar{S}_{1}$, the effective Lagrangian has only one contribution

$$
\mathcal{L}_{\text {eff }}=\sqrt{2} G_{F} c^{R R}\left(\bar{u}_{R} \gamma_{\mu} c_{R}\right)\left(\bar{\chi}_{R} \gamma^{\mu} \chi_{R}\right),
$$

with

$$
c^{R R}=\frac{v^{2}}{2 M_{\bar{S}_{1}}^{2}} \bar{y}_{1 c \chi}^{R R} \bar{y}_{1 u \chi}^{R R *} .
$$

In the case of $\tilde{R}_{2}$

$$
\mathcal{L}_{\text {eff }}=\sqrt{2} G_{F} c^{L R}\left(\bar{u}_{L} \gamma_{\mu} c_{L}\right)\left(\bar{\chi}_{R} \gamma^{\mu} \chi_{R}\right),
$$

with

$$
c^{L R}=-\frac{v^{2}}{2 M_{\tilde{R}_{2}}^{2}}\left(V \tilde{y}_{2}^{L R}\right)_{u \chi}\left(V \tilde{y}_{2}^{L R}\right)_{c \chi}^{*} .
$$

Note that in the case of $\tilde{R}_{2}$, the couplings $\tilde{y}_{2 j \chi}^{L R}$ for $j=d, s, b$ enter in the amplitudes $b \rightarrow s(d) \chi \bar{\chi}$ or $s \rightarrow d \chi \bar{\chi}$. However, it was found in Ref. [17] that the experimental rates for $K \rightarrow \pi \nu \bar{\nu}$ are very close to the SM rate [18], leaving very little room for NP contributions.

\section{III. $\bar{S}_{1}$ IN $c \rightarrow u \chi \bar{\chi}$}

Due to its quantum numbers, the colored scalar $\bar{S}_{1}$ and $\chi$ can interact only with up-like quarks. Most generally, the number of $\chi$ 's can be three and the matrix $y_{1 i j}^{R R}$ can have $9 \times 2$ parameters. Here, we consider one $\chi$, that can couple to both $u$ and $c$ quarks. These two couplings $\bar{y}_{1 c \chi}^{R R}$ and $\bar{y}_{1 u \chi}^{R R}$ might enter in amplitudes for processes with down-like quarks at the loop level, as discussed in [19]. Obviously, due to the right-handed nature of $\chi$, one can immediately see that in the case of $\bar{S}_{1}$, the effective Lagrangian has only the contribution

$$
\mathcal{L}_{\text {eff }}=\sqrt{2} G_{F} \frac{v^{2}}{2 M_{\bar{S}_{1}}^{2}} \bar{y}_{1 c \chi}^{R R} \bar{y}_{1 u \chi}^{R R *}\left(\bar{u}_{R} \gamma_{\mu} c_{R}\right)\left(\bar{\chi}_{R} \gamma^{\mu} \chi_{R}\right) .
$$

First, we discuss constraints from the $D^{0}-\bar{D}^{0}$ mixing and then consider the exclusive decays $D^{0} \rightarrow \chi \bar{\chi}, D^{0} \rightarrow \bar{\chi} \chi \gamma$, and $D \rightarrow \pi \chi \bar{\chi}$. The authors of Ref. [13] considered scalar leptoquarks allowing each up-quark coupling to a different flavor of lepton or right-handed neutrino. In such a way, they avoid constraints from the $D^{0}-\bar{D}^{0}$ mixing.

\section{A. Constraints from $D^{\mathbf{0}}-\bar{D}^{0}$}

The strongest constraints on $\chi$ interactions with $u$ and $c$ comes from the $D^{0}-\bar{D}^{0}$ oscillations. The interactions in Eqs. (2) and (3) can generate transition $D^{0}-\bar{D}^{0}$. Colored scalar $\bar{S}_{1}$ contributes to the operator entering the effective Lagrangian $[14,20]$

$$
\mathcal{L}_{\text {eff }}^{D \text { mix }}=-C_{6}\left(\bar{c} \gamma_{\mu} P_{R} u\right)\left(\bar{c} \gamma^{\mu} P_{R} u\right),
$$

with the Wilson coefficient given by

$$
C_{6}=\frac{1}{128 \pi^{2} M_{\bar{S}_{1}}^{2}}\left(\bar{y}_{1 c \chi}^{R R}\right)^{2}\left(\bar{y}_{1 u \chi}^{R R *}\right)^{2} .
$$

The standard way to write the hadronic matrix element is $\left\langle\bar{D}^{0}\left|\left(\bar{u} \gamma_{\mu} P_{R} c\right)\left(\bar{u} \gamma^{\mu} P_{R} c\right)\right| D^{0}\right\rangle=\frac{2}{3} m_{D}^{2} f_{D}^{2} B_{D}$, with the bag parameter $B_{D}(3 \mathrm{GeV})=0.757(27)(4)$ calculated in the minimal subtraction (MS) scheme, which was computed by the lattice QCD [21] and the $D$ meson decay constant defined as $\left.\langle 0| \bar{u} \gamma_{\mu} \gamma_{5} c\right)|D(p)\rangle=i f_{D} p_{\mu}$, with $f_{D}=$ $0.2120(0.7) \mathrm{GeV}[22,23]$. Due to large nonperturbative contributions, the SM contribution is not well known. Therefore, in the absence of $\mathrm{CP}$ violation $(\mathrm{C}$ is charge conjugation, $\mathrm{P}$ parity), the robust bound on the product of the couplings can be obtained by requiring that the mixing frequency should be smaller than the world average $x=$ $2\left|M_{12}\right| / \Gamma=\left(0.43_{-0.11}^{+0.10}\right) \%$ by HFLAV [24]. The bound on this Wilson coefficient can be derived following [19,25]

$$
\left|r C_{6}\left(M_{\bar{S}_{1}}\right)\right| \frac{2 m_{D} f_{D}^{2} B_{D}}{3 \Gamma_{D}}<x
$$

with a renormalization factor $r=0.76$ due to running $C_{6}$ from scale $M_{\bar{S}_{1}} \simeq 1.5 \mathrm{TeV}$ down to $3 \mathrm{GeV}$. One can derive $\left|C_{6}\right|<2.3 \times 10^{-13} \mathrm{GeV}^{-2}$ or

$$
\begin{aligned}
\left|\bar{y}_{1 c \chi}^{R R} \bar{y}_{1 u \chi}^{R R *}\right|<1.7 \times 10^{-5} M_{\bar{S}_{1}} / \mathrm{GeV} . \\
c^{R R}<\frac{0.518 \mathrm{GeV}}{M_{\bar{S}_{1}}(\mathrm{GeV})}
\end{aligned}
$$

We present the Yukawa couplings product determined from the Belle bound [8] and the bound from $D^{0}-\bar{D}^{0}$ oscillations in Fig. 1.

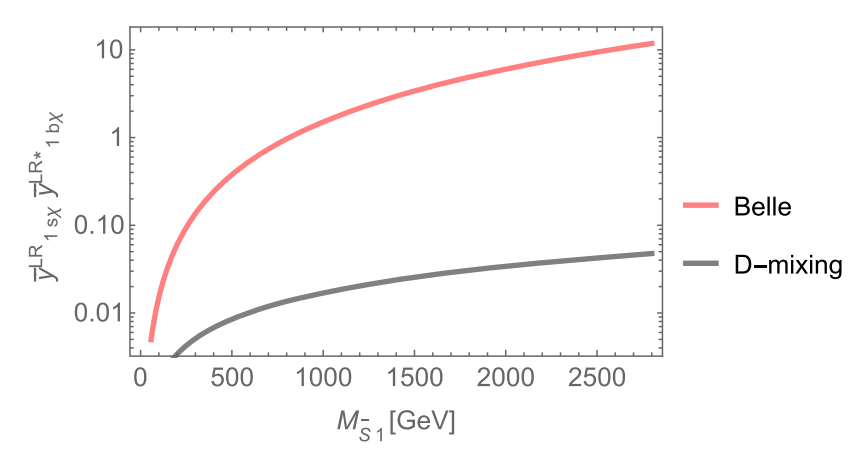

FIG. 1. The product of Yukawa couplings $\left|\bar{y}_{1 c \chi}^{R R} \bar{y}_{1 u \chi}^{R R *}\right|$ as a function of the $\bar{S}_{1}$ mass. The pink line denotes the bound derived from Belle result [8], while the gray one is obtained with the bound from $D^{0}-\bar{D}^{0}$ oscillations. 


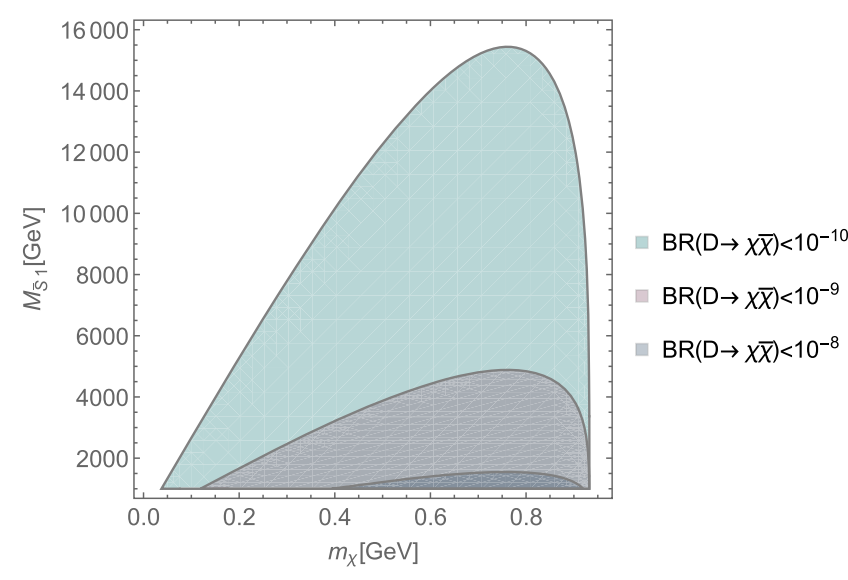

FIG. 2. The allowed mass region for $\bar{S}_{1}$ in the range $0<m_{\chi}<\left(m_{D}-m_{\pi}\right) / 2$. The regions are obtained assuming $\mathcal{B}\left(D^{0} \rightarrow \chi \bar{\chi}\right)<10^{-10}, \quad 10^{-9}$ and $10^{-8}$, for the product $\left|\bar{y}_{1 c \chi}^{R R} \bar{y}_{1 u \chi}^{R R *}\right|=1$.

\section{B. $D^{\mathbf{0}} \rightarrow \chi \bar{\chi}$}

The amplitude for this process can be written as

$\mathcal{M}\left(D^{0} \rightarrow \chi \bar{\chi}\right)=\frac{\sqrt{2}}{2} G_{F} f_{D} c^{R R} m_{\chi} \bar{u}_{\chi}\left(p_{1}\right) \gamma_{5} v_{\chi}\left(p_{2}\right)$,

leading to the branching ratio

$\mathcal{B}\left(D^{0} \rightarrow \chi \bar{\chi}\right)=\frac{1}{\Gamma_{D}} \frac{G_{F}^{2} f_{D}^{2} m_{D}}{16 \pi}\left|c^{R R}\right|^{2} m_{\chi}^{2} \sqrt{1-\frac{4 m_{\chi}^{2}}{m_{D}^{2}}}$.

Using Belle bound $\mathcal{B}\left(D^{0} \rightarrow \chi \bar{\chi}\right)<9.4 \times 10^{-5}$ [8], one can find easily the bound on Wilson coefficient $\left|c^{R R}\right|_{\text {Belle }}<0.046$. This value is derived for the mass $m_{\chi}=0.8 \mathrm{GeV}$. We analyze the dependence on the mass of $\bar{S}_{1}$, assuming the branching ratio for $\mathcal{B}\left(D^{0} \rightarrow \chi \bar{\chi}\right)<10^{-10}, 10^{-9}$ and $10^{-8}$, with $\left|\bar{y}_{1 c \chi}^{R R} \bar{y}_{1 u \chi}^{R R *}\right|=1$. We present our result in Fig. 2 and find that mass of $\bar{S}_{1}$, using these reasonable assumptions, can be within LHC reach. In Table II we show the upper bounds on the branching ratio $\mathcal{B}\left(D^{0} \rightarrow \chi \bar{\chi}\right)$ for three selected values of $m_{\chi}=0.2,0.5,0.8 \mathrm{GeV}$, using the constraints from the $D^{0}-\bar{D}^{0}$ mixing, $c^{R R} \leq 5.18 \times 10^{-4}$, with $M_{\bar{S}_{1}}=1000 \mathrm{GeV}$.

TABLE II. Branching ratios for $\mathcal{B}\left(D^{0} \rightarrow \chi \bar{\chi}\right)$ for three selected values of $m_{\chi}$. The constraints from the $D^{0}-\bar{D}^{0}$ mixing is used, with $c^{R R} \leq 5.18 \times 10^{-4}$, assuming $M_{\bar{S}_{1}}=1000 \mathrm{GeV}$.

\begin{tabular}{lc}
\hline \hline$m_{\chi}(\mathrm{GeV})$ & $\mathcal{B}\left(D^{0} \rightarrow \chi \bar{\chi}\right)_{D-\bar{D}}$ \\
\hline 0.2 & $<2.8 \times 10^{-9}$ \\
0.5 & $<1.5 \times 10^{-8}$ \\
0.8 & $<2.3 \times 10^{-8}$ \\
\hline \hline
\end{tabular}

\section{C. $D^{0} \rightarrow \chi \bar{\chi} \gamma$}

The authors of Ref. [2] suggested, that the helicity suppression, present in the $D^{0} \rightarrow \chi \bar{\chi}$ amplitude for $m_{\chi}=0$, is lifted by an additional photon in the final state and therefore $D^{0} \rightarrow \chi \bar{\chi} \gamma$ might bring additional information on detection of invisibles in the final state. They found that the branching decay is

$$
\mathcal{B}\left(D^{0} \rightarrow \chi \bar{\chi} \gamma\right)=\frac{G_{F}^{2} F_{D Q}^{2} f_{D}^{2}\left|c^{R R}\right|^{2} m_{D}^{2} \alpha}{1152 \pi^{2} \Gamma_{D} \sqrt{1-4 x_{\chi}^{2}}} Y\left(x_{\chi}\right) .
$$

In the above equations $x_{\chi}=m_{\chi} / m_{D}, \quad F_{D Q}=$ $2 / 3\left(-1 /\left(m_{D}-m_{c}\right)+1 / m_{c}\right), \quad f_{D}=0.2120(0.7) \mathrm{GeV}$ $[22,23]$ and $Y\left(x_{\chi}\right)$ is given in the Appendix. Coefficient $c^{R R}$ is constrained by Eq. (13). In Table III we give bounds on the branching ratio for $\mathcal{B}\left(D^{0} \rightarrow \chi \bar{\chi} \gamma\right)$ for three selected values of $m_{\chi}=0.2,0.5,0.8 \mathrm{GeV}$. Comparing these results with the SM result presented in Ref. [2] $\mathcal{B}\left(D^{0} \rightarrow \nu \bar{\nu} \gamma\right)_{\mathrm{SM}}=3.96 \times 10^{-14}$, we see that the existing Belle bound allows significant branching ratio. For massless $\chi$, the Belle limit on the Wilson coefficient is not applicable (15). For larger values of $m_{\chi}$, the bound from the $D^{0}-\bar{D}^{0}$ mixing leads to the branching ratio close to the $\mathrm{SM}$ results. Due to the mass of $\chi$, the photon energy can be in the range $0 \leq E_{\gamma} \leq\left(m_{D}^{2}-m_{\chi}^{2}\right) /\left(2 m_{D}\right)$, which in principle would distinguish the SM contribution from the contributions with massive invisible fermions.

\section{D. $\boldsymbol{D} \rightarrow \pi \chi \bar{\chi}$}

The rare charm decays due to GIM-mechanism cancellation are usually dominated by long distance contributions. Long distance contributions to the exclusive decay channel $D \rightarrow \pi \nu \bar{\nu}$ were considered in Ref. [26]. For example, the branching ratio $\operatorname{BR}\left(D^{+} \rightarrow \pi^{+} \rho^{0} \rightarrow \pi^{+} \nu \bar{\nu}\right)<5 \times 10^{-16}$. The authors of [26] discussed another possibility $D^{+} \rightarrow$ $\tau^{+} \nu \rightarrow \pi^{+} \bar{\nu} \nu$ and found that the branching ratio should be smaller than $1.8 \times 10^{-16}$. An interesting study of these effects was done in Ref. [27], implying that in order to

TABLE III. Bounds on the branching ratio for $\mathcal{B}\left(D^{0} \rightarrow \chi \bar{\chi} \gamma\right)$. In the second column we use, the constraint from the $D^{0}-\bar{D}^{0}$ mixing assuming $M_{\bar{S}_{1}}=1000 \mathrm{GeV}$. We use the Belle bound $\mathcal{B}\left(D^{0} \rightarrow \mathscr{E}\right)<9.4 \times 10^{-5}$ in the third column. With this bound the Wilson coefficient for $m_{\chi}=0$ cannot be fixed, reflecting in the third column's missing bound.

\begin{tabular}{lcc}
\hline \hline$m_{\chi}(\mathrm{GeV})$ & $\mathcal{B}\left(D^{0} \rightarrow \chi \bar{\chi} \gamma\right)_{D-\bar{D}}$ & $\mathcal{B}\left(D^{0} \rightarrow \chi \bar{\chi} \gamma\right)_{\text {Belle }}$ \\
\hline 0 & $<3.9 \times 10^{-12}$ & $\ldots$ \\
0.2 & $<3.0 \times 10^{-12}$ & $<1.3 \times 10^{-7}$ \\
0.5 & $<1.0 \times 10^{-12}$ & $<6.3 \times 10^{-9}$ \\
0.8 & $<5.4 \times 10^{-14}$ & $<2.2 \times 10^{-10}$ \\
\hline \hline
\end{tabular}


avoid these effects one should make cuts in the invariant $\chi \bar{\chi}$ mass square, $q_{\mathrm{cut}}^{2}<\left(m_{\tau}^{2}-m_{\pi}^{2}\right)\left(m_{D}^{2}-m_{\tau}^{2}\right) / m_{\tau}^{2}$.

The amplitude for $D \rightarrow \pi \chi \bar{\chi}$ can be written as

$$
\begin{aligned}
\mathcal{M}(D \rightarrow \pi \chi \bar{\chi})= & \sqrt{2} G_{F} c^{R R} \bar{u}_{\chi}\left(p_{1}\right) \gamma_{\mu} P_{R} v_{\chi}\left(p_{2}\right) \\
& \times\left\langle\pi(k)\left|\bar{u} \gamma^{\mu} P_{R} c\right| D(p)\right\rangle,
\end{aligned}
$$

with the standard form-factors definition

$$
\begin{aligned}
& \left\langle\pi(k)\left|\bar{u} \gamma^{\mu}\left(1 \pm \gamma_{5}\right) c\right| D(p)\right\rangle \\
& =F_{+}\left(q^{2}\right)\left[(p+k)^{\mu}-\frac{m_{D}^{2}-m_{\pi}^{2}}{q^{2}} q^{\mu}\right] \\
& \quad+F_{0}\left(q^{2}\right) \frac{m_{D}^{2}-m_{\pi}^{2}}{q^{2}} q^{\mu},
\end{aligned}
$$

with $q=p-k$. We follow the update of the form factors in Ref. [28]. Using their results, we write the amplitudes in the form given in Ref. [20]

$$
\begin{aligned}
\mathcal{M}\left(D(p) \rightarrow \pi(k) \chi\left(p_{1}\right) \bar{\chi}\left(p_{2}\right)\right) \\
=\frac{\sqrt{2}}{2} G_{F}\left[V\left(q^{2}\right) \bar{u}_{\chi}\left(p_{1}\right) \not p v_{\chi}\left(p_{2}\right)\right. \\
+A\left(q^{2}\right) \bar{u}_{\chi}\left(p_{1}\right) \not p \gamma_{5} v_{\chi}\left(p_{2}\right) \\
\left.\quad+P\left(q^{2}\right) \bar{u}_{\chi}\left(p_{1}\right) \gamma_{5} v_{\chi}\left(p_{2}\right)\right],
\end{aligned}
$$

with the following definitions

$$
\begin{aligned}
& V\left(q^{2}\right)=A\left(q^{2}\right) \equiv c^{R R} F_{+}\left(q^{2}\right), \\
& P\left(q^{2}\right) \equiv-c^{R R} m_{\chi}\left[F_{+}\left(q^{2}\right)-\frac{m_{D}^{2}-m_{\pi}^{2}}{q^{2}}\left(F_{0}\left(q^{2}\right)-F_{+}\left(q^{2}\right)\right)\right] .
\end{aligned}
$$

We can determine the differential decay rate distribution as

$$
\frac{d \mathcal{B}(D \rightarrow \pi \bar{\chi} \chi)}{d q^{2}}=\frac{1}{\Gamma_{D}} N \lambda^{1 / 2} \beta\left(q^{2}\right)\left[2 a\left(q^{2}\right)+\frac{2}{3} c\left(q^{2}\right)\right] .
$$

with notation $\lambda \equiv \lambda\left(m_{D}^{2}, m_{\pi}^{2}, q^{2}\right),\left(\lambda(x, y, z)=(x+y+z)^{2}-\right.$ $4(x y+y z+z x)), \quad \beta\left(q^{2}\right)=\sqrt{1-4 m_{\chi}^{2} / q^{2}} \quad$ and $\quad N=$ $G_{F}^{2} /\left(512 \pi^{3} m_{D}^{3}\right)$. There is a multiplication by two in the differential decay rate for the charged charm meson compared to neutral $D$.

The integration bounds should be $4 m_{\chi}^{2} \leq q^{2} \leq$ $\left(m_{D}-m_{\pi}\right)^{2}$ in the case of $m_{\chi}=0.5,0.8 \mathrm{GeV}$, while for masses $m_{\chi}=0.2 \mathrm{GeV}$ and smaller, $q_{\mathrm{cut}}^{2}$ is used from Ref. [27]. The corresponding mass of the invisible fermion is $m_{\chi} \geq \sqrt{q_{\text {cut }}^{2} / 4} \simeq 0.29 \mathrm{GeV}$. This enables us to avoid the region in which the effects of the long distance dynamics

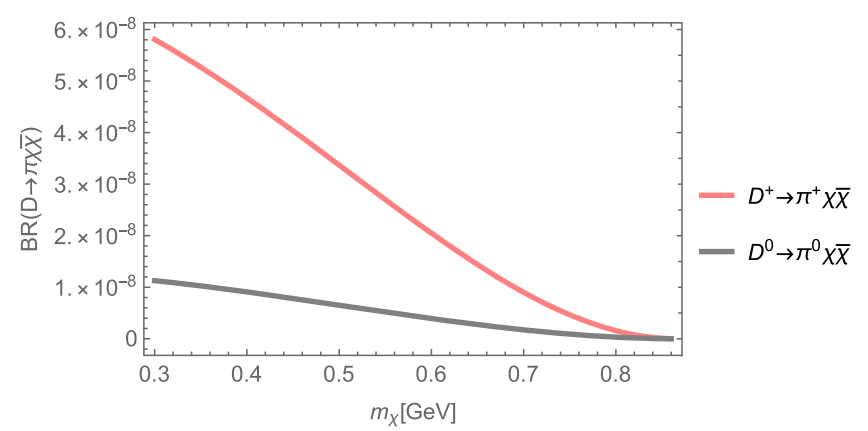

FIG. 3. Branching fraction for $D^{+} \rightarrow \pi^{+} \chi \bar{\chi}$ and $D^{0} \rightarrow \pi^{0} \chi \bar{\chi}$ as a function of $m_{\chi}$.

dominates. One can use the Belle bound [8] for $\mathcal{B}(D \rightarrow \not E)$ and determine $c^{R R}$ from $D^{0} \rightarrow \chi \bar{\chi}$ for each $\chi$ mass. We obtain $\mathcal{B}\left(D^{0} \rightarrow \pi^{0} \chi \bar{\chi}\right)_{\text {Belle }} \leq 4.0 \times 10^{-4}, 4.1 \times 10^{-5}$, $1.2 \times 10^{-6}$ and $\mathcal{B}\left(D^{+} \rightarrow \pi^{+} \chi \bar{\chi}\right)_{\text {Belle }} \leq 2.0 \times 10^{-3}$, $2.1 \times 10^{-4}, 6.1 \times 10^{-6}$ for $m_{\chi}=0.2,0.5,0.8 \mathrm{GeV}$ respectively. In Fig. 3, we present branching ratios for the allowed range of the $\chi$ mass. The current Belle bound used in the Wilson coefficient leads to the significant increase of the branching ratios for both decay modes which may be possible to observe at the future tau-charm factories and Belle II experiment. However, this bound is relatively weak comparing it with the bound coming from the $D^{0}-\bar{D}^{0}$ oscillations. We calculate branching ratios using the constraint from the $D^{0}-\bar{D}^{0}$ mixing. In Table IV, we present our predictions for the upper bound on the $\mathcal{B}(D \rightarrow \pi \chi \bar{\chi})$, assuming the mass of $M_{\bar{S}_{1}}=1000 \mathrm{GeV}$ and taking the $m_{\chi}=0,0.2,0.5,08 \mathrm{GeV}$. In the case $m_{\chi}=0.2 \mathrm{GeV}$, the cut in integration variable is done by taking $q_{\text {cut }}^{2}$, as described above.

\section{IV. $\tilde{\boldsymbol{R}}_{\mathbf{2}}$ IN $c \rightarrow \boldsymbol{u} \chi \bar{\chi}$}

The $\tilde{R}_{2}$ leptoquark is a weak doublet and it interacts with quark doublets (3). This feature increases the possibility to constrain the Yukawa couplings $\tilde{y}_{2 \tilde{d} x}^{L R}$ with the down-like quark $\tilde{d}=d, s, b$. The simplest scenario is to assume that only one of these couplings is nonzero. It contributes to the Wilson coefficient in (7) leading to

TABLE IV. Branching ratios for $\mathcal{B}(D \rightarrow \pi \chi \bar{\chi})$. In the second and the third columns the constraint from the $D^{0}-\bar{D}^{0}$ mixing is used, assuming the mass of $M_{\bar{S}_{1}}=1000 \mathrm{GeV}$. In the case $m_{\chi}=0.2 \mathrm{GeV}$, the cut in the integration variable is done by taking $q_{\text {cut }}^{2}$, as described in the text.

\begin{tabular}{lcc}
\hline \hline$m_{\chi}(\mathrm{GeV})$ & $\mathcal{B}\left(D^{0} \rightarrow \pi^{0} \chi \bar{\chi}\right)_{D-\bar{D}}$ & $\mathcal{B}\left(D^{+} \rightarrow \pi^{+} \chi \bar{\chi}\right)_{D-\bar{D}}$ \\
\hline 0 & $<1.5 \times 10^{-8}$ & $<5.8 \times 10^{-8}$ \\
0.2 & $<1.2 \times 10^{-8}$ & $<6.1 \times 10^{-8}$ \\
0.5 & $<6.6 \times 10^{-9}$ & $<3.3 \times 10^{-8}$ \\
0.8 & $<3.0 \times 10^{-10}$ & $<1.5 \times 10^{-9}$ \\
\hline \hline
\end{tabular}




$$
c^{L R}=-\frac{v^{2}}{2 M_{\tilde{R}_{2}}^{2}} V_{u s} V_{c s}^{*}\left|\tilde{y}_{2 s \chi}^{L R}\right|^{2}
$$

if we consider only one nonzero coupling e.g., $\tilde{y}_{2 s \chi}^{L R}$. Lowenergy data for $\Phi(1020)$ spin-one meson have, according to [23], the upper bound $\mathcal{B}(\Phi \rightarrow$ invisibles $)<1.7 \times 10^{-4}$, which is not restrictive enough. The $D^{0}-\bar{D}^{0}$ oscillations are more restrictive, and using the condition in (12), we derive

$$
\left.V_{u s} V_{c s}^{*}\left|\tilde{y}_{2 s \chi}^{L R}\right|^{2}\right|_{D-\bar{D}}<1.7 \times 10^{-5} M_{\tilde{R}_{2}} / \mathrm{GeV} .
$$

One can instead assume that either $\tilde{y}_{2 d \chi}^{L R}$ or $\tilde{y}_{2 b \chi}^{L R}$ are nonzero, while others are set to zero. Simple replacement in the CKM matrix elements $s \rightarrow d(b)$ should be done too. This assumption will imply the same results already presented for the $\bar{S}_{1}$ mediation in the previous section.

In the second scenario we can set the $\tilde{y}_{2 s \chi}^{L R}$ and $\tilde{y}_{2 b \chi}^{L R}$ couplings to be nonzero. We can constrain them by the bound on the branching ratio $B \rightarrow K \chi \bar{\chi}$ decays and the $B_{s}-\bar{B}_{s}$ mixing. The charm decays allow mass of $m_{\chi} \leq\left(m_{D}-m_{\pi}\right) / 2$. We consider the most constraining bounds coming from decays $B \rightarrow K E$ and from the oscillations of $B_{s}-\bar{B}_{s}$. In order to avoid very strong constraints from $K \rightarrow \pi \nu \bar{\nu}$, we limit ourselves to the scenario $\tilde{y}_{2 d \chi}^{L R} \simeq 0$. The decay $B \rightarrow K \mathscr{E}$ was recently studied by the authors of Ref. [29]. They pointed out that current bound on the rate $B \rightarrow K \mathscr{E}$ when the SM branching ratio for $B \rightarrow K \nu \bar{\nu}$ is subtracted from the experimental bound on $\mathcal{B}\left(B^{+} \rightarrow K^{+} \mathscr{E}\right)$ is the most constraining. They derived $\mathcal{B}(B \rightarrow K \mathscr{E})<9.7 \times 10^{-6}$ as the strongest bound among $B \rightarrow H_{s} E$ ( $H_{s}$ is a hadron containing the $s$ quark).

\section{A. Constraints from $B \rightarrow K E$ and $B_{s}-\bar{B}_{s}$ oscillations}

The amplitude for $B \rightarrow K \chi \bar{\chi}$ can be written as

$$
\begin{aligned}
\mathcal{M}(B \rightarrow K \chi \bar{\chi})= & \sqrt{2} G_{F} c_{B}^{L R} \bar{u}_{\chi}\left(p_{1}\right) \gamma_{\mu} P_{R} v_{\chi}\left(p_{2}\right) \\
& \times\left\langle K(k)\left|\bar{s} \gamma^{\mu} P_{L} b\right| B(p)\right\rangle .
\end{aligned}
$$

In the case of Wilson coefficient $c_{B}^{L R}$ it is easy to find [14]

$$
c_{B}^{L R}=-\frac{v^{2}}{2 M_{\tilde{R}_{2}}^{2}} \tilde{y}_{2 b \chi}^{L R} \tilde{y}_{2 s \chi}^{L R *} .
$$

The integration over the phase space depends on the mass of $m_{\chi}$ we chose. Here we choose a mass of $\chi$, which we used in $D$ decays $0<m_{\chi}<\left(m_{D}-m_{\pi}\right) / 2$. The bounds on the Wilson coefficient in Eq. (25) are the following: $\left|c_{B}^{L R}\right|<3.3 \times 10^{-4},<4.9 \times 10^{-4}$, and $<9.1 \times 10^{-4}$ for $m_{\chi}=0.2,0.5,0.8 \mathrm{GeV}$.

There are two box diagrams with $\chi$ within the box contributing to the $B_{s}-\bar{B}_{s}$ oscillations. The contribution of
$\tilde{R}_{2}$ box diagrams to the effective Lagrangian for the $B_{s}-\bar{B}_{s}$ oscillation is

$$
\begin{aligned}
\mathcal{L}_{\Delta B=2}^{N P}=- & \frac{1}{128 \pi^{2}} \frac{\left(\tilde{y}_{2 s \chi}^{L R}\right)^{2}\left(\tilde{y}_{2 b \chi}^{L R *}\right)^{2}}{M_{\tilde{R}_{2}}^{2}} \\
& \times\left(\bar{s} \gamma_{\mu} P_{R} b\right)\left(\bar{s} \gamma^{\mu} P_{R} b\right) .
\end{aligned}
$$

We can understand this result in terms of the recent study of new physics in the $B_{s}-\bar{B}_{s}$ oscillation in [30]. The authors of [30] introduced the following notation of the new physics contribution containing the right-handed operators as

$\mathcal{L}_{\Delta B=2}^{N P} \supset-\frac{4 G_{F}}{\sqrt{2}}\left(V_{t b} V_{t s}^{*}\right)^{2} C_{b s}^{R R}\left(\bar{s} \gamma_{\mu} P_{R} b\right)\left(\bar{s} \gamma^{\mu} P_{R} b\right)$.

Following their notation, one can write the modification of the SM contribution by the NP as in Ref. [30]

$$
\frac{\Delta M_{s}^{\mathrm{SM}+N P}}{\Delta M_{s}^{\mathrm{SM}}}=\left|1+\frac{\eta^{6 / 23}}{R_{\text {loop }}^{\mathrm{SM}}} C_{b s}^{R R}\right| .
$$

They found that $R_{\text {loop }}^{\text {SM }}=(1.31 \pm 0.010) \times 10^{-3}$ and $\eta=\alpha_{s}\left(\mu_{N P}\right) / \alpha_{s}\left(\mu_{b}\right)$. Relying on the lattice QCD results of the two collaborations FNAL/MILC [31], and HPQCD [32], the FLAG averaging group [22] published the following results which we use in our calculations

$\Delta M_{s}^{\mathrm{FLAG} 2019}=\left(20.1_{-1.6}^{+1.2}\right) \mathrm{ps}^{-1}=\left(1.13_{-0.09}^{+0.07}\right) \Delta M_{s}^{\mathrm{exp}}$.

From these results, one can easily determine the bound

$$
\left|\frac{\left(\tilde{y}_{2 s \chi}^{L R}\right)^{2}\left(\tilde{y}_{2 b \chi}^{L R *}\right)^{2}}{M_{\tilde{R}_{2}}^{2}}\right| \leq 1.39 \times 10^{-8} \mathrm{GeV}^{-2},
$$

The same couplings $\tilde{y}_{2 s \chi}^{L R} \tilde{y}_{2 b \chi}^{L R *}$ enter in the $D^{0}-\bar{D}^{0}$ mixing (9) and condition (12), and one can derive

$$
\begin{aligned}
& {\left.\left[\left(V_{u s} \tilde{y}_{2 s \chi}^{L R}\right)\left(V_{c b} \tilde{y}_{2 b \chi}^{L R}\right)^{*}+\left(V_{c s} \tilde{y}_{2 s \chi}^{L R}\right)\left(V_{u b} \tilde{y}_{2 b \chi}^{L R}\right)^{*}\right]\right|_{D-\bar{D}}} \\
& \quad<1.7 \times 10^{-5} M_{\tilde{R}_{2}} / \mathrm{GeV} .
\end{aligned}
$$

The bound on coefficients in (30) lead to the one order of magnitude stronger constraint than the one in (31), $\tilde{y}_{2 s \chi}^{L R} \tilde{y}_{2 b \chi}^{L R *}<1.58 \times 10^{-6} M_{\tilde{R}_{2}} / \mathrm{GeV}$. In our numerical calculations we use this bound and do not specify the mass of $\tilde{R}_{2}$. However, one can combine these constraints and determined the $\tilde{R}_{2}$ mass, which can satisfy both conditions. In Fig. 4 we present dependence of the couplings $\tilde{y}_{2 s \chi}^{L R} \tilde{y}_{2 b \chi}^{L R *}$ as a function of mass $M_{\tilde{R}_{2}}$ for masses using constraints from $B_{s}^{0}-\bar{B}_{s}^{0}$ mixing and from the bound $\mathcal{B}\left(B^{+} \rightarrow K^{+} \not E\right)<$ $9.7 \times 10^{-6}$ for $m_{\chi}=0.2,0.5,0.8 \mathrm{GeV}$. From Fig. 4 we see 


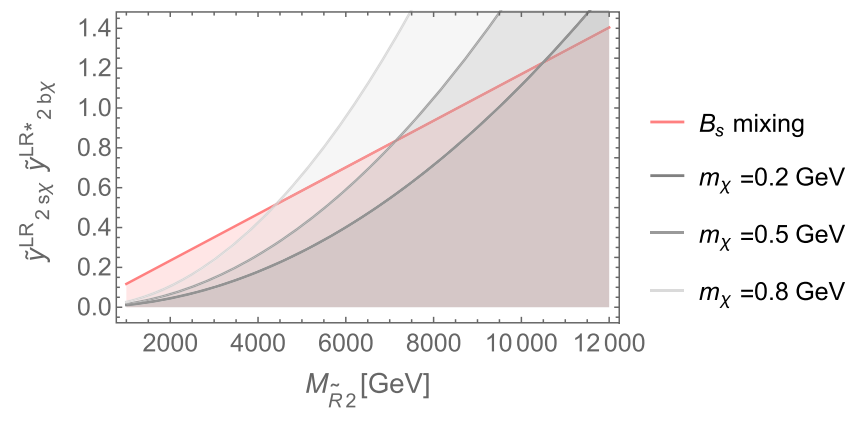

FIG. 4. The allowed mass for $\tilde{R}_{2}$. Constraints are derived from the $B_{s}^{0}-\bar{B}_{s}^{0}$ mixing and from the bound $\mathcal{B}\left(B^{+} \rightarrow K^{+} \mathscr{E}\right)<9.7 \times$ $10^{-6}$ for $m_{\chi}=0.2,0.5,0.8 \mathrm{GeV}$.

that the largest mass of $\tilde{R}_{2}$, which satisfies both conditions is $M_{\tilde{R}_{2}} \simeq 10800,7100,4400 \mathrm{GeV}$ for the masses $m_{\chi}=0.2$, $0.5,0.8 \mathrm{GeV}$ respectively. All $\tilde{R}_{2}$ masses below these limiting values are allowed, and interestingly, they are within LHC reach [33-36].

$$
\begin{gathered}
\text { B. } \tilde{\boldsymbol{R}}_{\mathbf{2}} \text { in } \mathcal{B}\left(D^{\mathbf{0}} \rightarrow \chi \bar{\chi}\right), \mathcal{B}\left(D^{\mathbf{0}} \rightarrow \chi \bar{\chi} \gamma\right) \\
\text { and } \mathcal{B}\left(D^{+} \rightarrow \boldsymbol{\pi}^{+} \chi \bar{\chi}\right)
\end{gathered}
$$

Using the same expressions as in the previous section, we calculate branching ratios for $D^{0} \rightarrow \chi \bar{\chi}, D^{0} \rightarrow \chi \bar{\chi} \gamma$ and present them in Table $\mathrm{V}$. The results for $D \rightarrow \pi \chi \bar{\chi}$ are presented in Table VI. The Wilson coefficient $c_{D}^{L R}$ is obtained using the constraint from $B \rightarrow K \mathscr{E}$. For $m_{\chi}=0.2,0.5,0.8 \mathrm{GeV}$ they are $c_{D}^{L R}=\mid\left(V_{u s} V_{c b}^{*}+V_{c s}\right.$ $\left.V_{u b}^{*}\right) c_{B}^{L R} \mid=4.4 \times 10^{-6}, 6.6 \times 10^{-6}, 1.2 \times 10^{-5}$. Compared

TABLE V. Branching ratios for $\mathcal{B}\left(D^{0} \rightarrow \chi \bar{\chi}\right)$ and $\mathcal{B}\left(D^{0} \rightarrow \chi \bar{\chi} \gamma\right)$. The bounds on the Wilson coefficient $c_{D}^{L R}$ derived from the $\mathcal{B}(B \rightarrow K E)<9.7 \times 10^{-6}$ for selected masses of $\chi$.

\begin{tabular}{lll}
\hline \hline$m_{\chi}(\mathrm{GeV})$ & $\mathcal{B}\left(D^{0} \rightarrow \chi \bar{\chi}\right)$ & $\mathcal{B}\left(D^{0} \rightarrow \chi \bar{\chi} \gamma\right)$ \\
\hline 0.2 & $<2.2 \times 10^{-13}$ & $<2.0 \times 10^{-15}$ \\
0.5 & $<2.5 \times 10^{-12}$ & $<1.4 \times 10^{-15}$ \\
0.8 & $<1.1 \times 10^{-11}$ & $<2.1 \times 10^{-16}$ \\
\hline \hline
\end{tabular}

TABLE VI. Branching ratios for $\mathcal{B}\left(D^{0} \rightarrow \pi^{0} \chi \bar{\chi}\right)$ and $\mathcal{B}\left(D^{+} \rightarrow \pi^{+} \chi \bar{\chi}\right)$. The bounds on the Wilson coefficient $c_{D}^{L R}$ derived from $\mathcal{B}(B \rightarrow K \mathscr{E})<9.7 \times 10^{-6}$. In the case $m_{\chi}=0.2 \mathrm{GeV}$, the cut in the integration variable is done by taking $q_{\text {cut }}^{2}$, as described in the text.

\begin{tabular}{lll}
\hline \hline$m_{\chi}(\mathrm{GeV})$ & $\mathcal{B}\left(D^{0} \rightarrow \pi^{0} \chi \bar{\chi}\right)$ & $\mathcal{B}\left(D^{+} \rightarrow \pi^{+} \chi \bar{\chi}\right)$ \\
\hline 0.2 & $<9.4 \times 10^{-13}$ & $<4.8 \times 10^{-12}$ \\
0.5 & $<1.1 \times 10^{-12}$ & $<5.4 \times 10^{-12}$ \\
0.8 & $<1.3 \times 10^{-13}$ & $<6.9 \times 10^{-13}$ \\
\hline \hline
\end{tabular}

with the colored scalar $\bar{S}_{1}$ mediation, the branching ratios for all three decay modes are suppressed for several orders of magnitude, indicating the important role of constraints from $B$ mesons. Such suppressed branching ratios of all the rare charm decays mediated by $\tilde{R}_{2}$ is almost impossible to observe. On the other hand, decays of hadrons containing $b$ quarks, mediated by $\tilde{R}_{2}$ are much more suitable for searches of invisible fermions.

\section{SUMMARY AND OUTLOOK}

We have presented a study on rare charm decays with invisible massive fermions $\chi$ in the final state. We considered two cases with colored scalar mediators of the upquarks interaction with $\chi$. The simplest model is one with $\bar{S}_{1}=(\overline{3}, 1,-2 / 3)$, which couples only to weak up-quark singlets, and the second mediator is $\tilde{R}_{2}=(\overline{3}, 2,1 / 6)$ which couples to weak quark doublets.

In the case of $\bar{S}_{1}$, the relevant constraint comes from the $D^{0}-\bar{D}^{0}$ oscillations. We have calculated branching ratios for $D^{0} \rightarrow \chi \bar{\chi}, D^{0} \rightarrow \chi \bar{\chi} \gamma$ and $D \rightarrow \pi \chi \bar{\chi}$. The charm meson mixing severely constrain the branching ratio $D^{0} \rightarrow \chi \bar{\chi}$ in comparison with the experimental result for the branching ratio of $D^{0} \rightarrow \mathbb{E}$. For our choice of $m_{\chi}$ the branching ratio for $D^{0} \rightarrow \chi \bar{\chi} \gamma$ can be calculated using experimental bounds on the rate for $D^{0} \rightarrow \mathscr{E}$. In this case, there is an enhancement factor of up to three orders of magnitude, depending on the mass of $\chi$ in comparison with the constraints from the $D^{0}-\bar{D}^{0}$ oscillations. The branching ratios for $D \rightarrow \pi \chi \bar{\chi}$, based on charm mixing constraint, are of the order $10^{-8}$, suitable for searches at future tau-charm factories. BES III and Belle II experiments can reach sensitivities of the order $10^{-6}$.

The $\tilde{R}_{2}$ leptoquark offers more possibilities to fix the Yukawa couplings. The simplest scenario is to assume only one nonzero coupling of a down-like quark to $\chi$. In such scenario all predicted branching ratios for $D^{0} \rightarrow \chi \bar{\chi}, D^{0} \rightarrow$ $\chi \bar{\chi} \gamma$ and $D \rightarrow \pi \chi \bar{\chi}$ are the same as in the case $\bar{S}_{1}$. If two Yukawa couplings $\tilde{y}_{2 s \chi}^{L R}$ and $\tilde{y}_{2 b \chi}^{L R}$ are nonzero, we rely on constraints coming from $B(B \rightarrow K \mathscr{E})$ and from the $B_{s}^{0}-\bar{B}_{s}^{0}$ mixing. We find that the all three decay modes $D^{0} \rightarrow \chi \bar{\chi}$, $D^{0} \rightarrow \chi \bar{\chi} \gamma$ and $D^{+} \rightarrow \pi^{+} \chi \bar{\chi}$ now have branching ratios of a factor of three to four orders of magnitude smaller than in the case of colored scalar $\bar{S}_{1}$ mediation, making them very difficult to observe.

\section{ACKNOWLEDGMENTS}

The work of S. F. was in part financially supported by the Slovenian Research Agency (research core funding No. P10035). The work of A. N, was partially supported by the Advanced Grant of European Research Council (ERC) 884719 FAIME. 


\section{APPENDIX}

\section{Phase space factors}

In Eq. (16) phase space function $Y\left(x_{\chi}\right)$ is used

$$
\begin{aligned}
Y\left(x_{\chi}\right)= & 1-2 x_{\chi}^{2}+3 x_{\chi}^{2}\left(3-6 x_{\chi}^{2}+4 x_{\chi}^{4}\right) \sqrt{1-4 x_{\chi}^{2}} \\
& \times \log \left(\frac{2 x_{\chi}}{1+\sqrt{1-4 x_{\chi}^{2}}}\right)-11 x_{\chi}^{4}+12 x_{\chi}^{6} .
\end{aligned}
$$

In Eq. (21) $a\left(q^{2}\right)$ and $c\left(q^{2}\right)$ are introduced denoting

$$
\begin{aligned}
a\left(q^{2}\right)= & \frac{\lambda}{2}\left(\left|V\left(q^{2}\right)\right|^{2}+\left|A\left(q^{2}\right)\right|^{2}\right)+8 m_{\chi}^{2} m_{D}^{2}\left|A\left(q^{2}\right)\right|^{2} \\
& +2 q^{2}\left|P\left(q^{2}\right)\right|^{2}+4 m_{\chi}\left(m_{D}^{2}-m_{\pi}^{2}+q^{2}\right) \\
& \times \operatorname{Re}\left[A\left(q^{2}\right) P\left(q^{2}\right)^{*}\right], \\
c\left(q^{2}\right)= & -\frac{\lambda \beta^{2}}{2}\left(\left|V\left(q^{2}\right)\right|^{2}+\left|A\left(q^{2}\right)\right|^{2}\right) .
\end{aligned}
$$

\section{2. $D \rightarrow \pi$ form factors}

Following [37] one can use the $z$ expansion with

$$
z=\frac{\sqrt{t_{+}-q^{2}}-\sqrt{t_{+}-t_{0}}}{\sqrt{t_{+}-q^{2}}+\sqrt{t_{+}-t_{0}}},
$$

where $\quad t_{+}=\left(m_{D}+m_{\pi}\right)^{2} \quad$ and $\quad t_{0}=\left(m_{D}+m_{\pi}\right) \times$ $\left(\sqrt{m}_{D}-\sqrt{m}_{\pi}\right)$. The form factors can be written as
TABLE VII. Fit parameters for $F_{0}, F_{+}$in the $z$-series expansion for $D \rightarrow \pi$ [37].

\begin{tabular}{lrrrr}
\hline \hline$f(0)$ & $c_{+}$ & $P_{V}(\mathrm{GeV})^{-2}$ & $c_{0}$ & $P_{S}(\mathrm{GeV})^{-2}$ \\
\hline $\begin{array}{c}0.6117 \\
(354)\end{array}$ & $\begin{array}{r}-1.985 \\
(347)\end{array}$ & $\begin{array}{c}0.1314 \\
(127)\end{array}$ & $\begin{array}{r}-1.188 \\
(256)\end{array}$ & $\begin{array}{r}0.0342 \\
(122)\end{array}$ \\
\hline \hline$F_{+}^{D \rightarrow \pi}\left(q^{2}\right)=\frac{f^{D \rightarrow \pi}(0)+c_{+}^{D \rightarrow \pi}\left(z-z_{0}\right)\left(1+\frac{1}{2}\left(z+z_{0}\right)\right)}{1-P_{V} q^{2}}$,
\end{tabular}

$$
F_{0}^{D \rightarrow \pi}\left(q^{2}\right)=\frac{f^{D \rightarrow \pi}(0)+c_{0}^{D \rightarrow \pi}\left(z-z_{0}\right)\left(1+\frac{1}{2}\left(z+z_{0}\right)\right)}{1-P_{S} q^{2}},
$$

where $z_{0}=z\left(0, t_{0}\right)$. The fit parameters are given in Table VII. For the most recent discussion on form factors (see also [38]).

\section{3. $B \rightarrow K$ form factors}

Most recent results are presented in FLAG report [22]

$$
\begin{gathered}
F_{+}^{\mathrm{BK}}\left(q^{2}\right)=\frac{r_{1}}{1-\frac{q^{2}}{m_{R}^{2}}}+\frac{r_{2}}{1-\frac{q^{2}}{m_{R}^{2}}}, \\
F_{0}^{\mathrm{BK}}\left(q^{2}\right)=\frac{r_{1}}{1-\frac{q^{2}}{m_{R}^{\prime 2}}} .
\end{gathered}
$$

The parameters are $r_{1}=0.162, \quad r_{2}=0.173, \quad m_{R}=$ $5.41 \mathrm{GeV}$ and $m_{R^{\prime}}=6.12 \mathrm{GeV}$, as in [22].
[1] R. Bause, H. Gisbert, M. Golz, and G. Hiller, Phys. Rev. D 103, 015033 (2021).

[2] A. Badin and A. A. Petrov, Phys. Rev. D 82, 034005 (2010).

[3] B. Bhattacharya, C. M. Grant, and A. A. Petrov, Phys. Rev. D 99, 093010 (2019).

[4] E. Kou et al. (Belle-II Collaboration), Prog. Theor. Exp. Phys. (2019), 123C01; (2020), 029201(E).

[5] M. Ablikim et al. (BESIII Collaboration), Chin. Phys. C 44, 040001 (2020).

[6] A. Abada et al. (FCC Collaboration), Eur. Phys. J. C 79, 474 (2019).

[7] A. Abada et al. (FCC Collaboration), Eur. Phys. J. Special Topics 228, 261 (2019).

[8] Y. T. Lai et al. (Belle Collaboration), Phys. Rev. D 95, 011102 (2017).

[9] A. M. Sirunyan et al. (CMS Collaboration), Phys. Rev. D 98, 032005 (2018).
[10] R. Bause, H. Gisbert, M. Golz, and G. Hiller, Phys. Rev. D 101, 115006 (2020).

[11] E. Golowich, J. Hewett, S. Pakvasa, and A. A. Petrov, Phys. Rev. D 79, 114030 (2009).

[12] J. Martin Camalich, M. Pospelov, P. N. H. Vuong, R. Ziegler, and J. Zupan, Phys. Rev. D 102, 015023 (2020).

[13] G. Faisel, J. Y. Su, and J. Tandean, J. High Energy Phys. 04 (2021) 246.

[14] I. Doršner, S. Fajfer, A. Greljo, J. F. Kamenik, and N. Košnik, Phys. Rep. 641, 1 (2016).

[15] M. Bordone, C. Cornella, J. Fuentes-Martín, and G. Isidori, J. High Energy Phys. 10 (2018) 148.

[16] L. Di Luzio, A. Greljo, and M. Nardecchia, Phys. Rev. D 96, 115011 (2017).

[17] E. Cortina Gil et al. (NA62 Collaboration), arXiv:2103.15389.

[18] A. J. Buras, D. Buttazzo, J. Girrbach-Noe, and R. Knegjens, J. High Energy Phys. 11 (2015) 033.

[19] S. Fajfer and D. Susič, Phys. Rev. D 103, 055012 (2021). 
[20] S. Fajfer and N. Košnik, Eur. Phys. J. C 75, 567 (2015).

[21] N. Carrasco et al. (ETM Collaboration), Phys. Rev. D 92, 034516 (2015).

[22] S. Aoki et al. (Flavor Lattice Averaging Group), Eur. Phys. J. C 80, 113 (2020).

[23] P. A. Zyla et al. (Particle Data Group), Prog. Theor. Exp. Phys. (2020), 083C01.

[24] Y. S. Amhis et al. (HFLAV Collaboration), Eur. Phys. J. C 81, 226 (2021).

[25] S. Fajfer and N. Kosnik, Phys. Rev. D 79, 017502 (2009).

[26] G. Burdman, E. Golowich, J. L. Hewett, and S. Pakvasa, Phys. Rev. D 66, 014009 (2002).

[27] J. F. Kamenik and C. Smith, Phys. Lett. B 680, 471 (2009).

[28] R. Fleischer, R. Jaarsma, and G. Koole, Eur. Phys. J. C 80, 153 (2020).

[29] G. Li, T. Wang, Y. Jiang, J. B. Zhang, and G. L. Wang, Phys. Rev. D 102, 095019 (2020).

[30] L. Di Luzio, M. Kirk, A. Lenz, and T. Rauh, J. High Energy Phys. 12 (2019) 009.
[31] A. Bazavov et al. (Fermilab Lattice and MILC Collaborations), Phys. Rev. D 93, 113016 (2016).

[32] R. J. Dowdall, C. T. H. Davies, R. R. Horgan, G. P. Lepage, C. J. Monahan, J. Shigemitsu, and M. Wingate, Phys. Rev. D 100, 094508 (2019).

[33] B. Pascual-Dias, P. Saha, and D. London, J. High Energy Phys. 07 (2020) 144.

[34] G. F. Giudice, B. Gripaios, and R. Sundrum, J. High Energy Phys. 08 (2011) 055.

[35] A. M. Sirunyan et al. (CMS Collaboration), J. High Energy Phys. 08 (2018) 130.

[36] A. M. Sirunyan et al. (CMS Collaboration), J. High Energy Phys. 05 (2020) 033.

[37] V. Lubicz et al. (ETM Collaboration), Phys. Rev. D 96, 054514 (2017); 99, 099902(E) (2019); 100, 079901(E) (2019).

[38] D. Bečirević, F. Jaffredo, A. Peñuelas, and O. Sumensari, J. High Energy Phys. 05 (2021) 175. 